\title{
Patients should be extubated in the operating room after routine cardiac surgery: An inconvenient truth
}

\author{
Heather K. Hayanga, MD, MPH, ${ }^{a}$ Matthew B. Ellison, MD, ${ }^{a}$ and Vinay Badhwar, MD $^{\mathrm{b}}$
}

Feature Editor's Introduction-Although 24-hour ventilator times have remained the quality metric for Society for Thoracic Surgeons (STS) Star rankings, it has become apparent over the past several years that the association between vent time and morbidity occurs much earlier. ${ }^{1}$ The reporting of a 6-hour metric for the past 20 years in the STS Adult Cardiac Surgery Database (ACSD) was a recognition of its benefits ${ }^{2}$ and pushed $u s$ to earlier extubations, such that ventilation time $<6$ hours has doubled since 2005, currently being accomplished in $>60 \%$ of isolated coronary artery bypass grafting (CABG) surgeries (2017-20 ACSD Report).

In this month's JTCVS Techniques, Hayanga and colleagues $^{3}$ in their expert opinion are now challenging us to improve the quality of care we give our patients by raising the bar even higher to extubate patients before leaving the operating room, an event that currently occurs in fewer than 1 in 20 recipients of isolated $C A B G$, for example (2017-20 ACSD Report).

Their opinion that this will be better for our patients stands to reason, as this group has championed operating room (OR) extubations for almost a decade, and they substantiate their prejudice by citing their own experience. The authors point out that their propensitymatched study comparing OR extubations to early intensive care unit (ICU) extubations showed significant decreases in ICU length of stay (3 hours) and postoperative length of stay (1 day), and a cost savings of $\$ 8$ this should read $\$ 800$ to $\$ 900$ per patient. However, this expert opinion, which rests so heavily on this one study, might not sufficiently stress its limitations-that it suffers from being observational, retrospective, singleinstitutional, and clearly open to the criticism that variables not included in the multivariate analysis could have changed the results. Nevertheless, any of us in

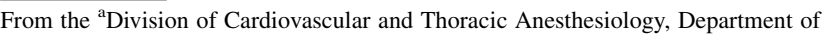
Anesthesiology and ${ }^{\mathrm{b}}$ Department of Cardiovascular and Thoracic Surgery, West Virginia University, Morgantown, WVa.

Read at the 100th Annual Meeting of The American Association for Thoracic Surgery: A Virtual Learning Experience, May 22-23, 2020.

Received for publication March 22, 2021; accepted for publication March 22, 2021; available ahead of print April 24, 2021

Address for reprints: Vinay Badhwar, MD, Department of Cardiovascular and Thoracic Surgery, West Virginia University, 1 Medical Center Dr, Morgantown, WV 26506-8059 (E-mail: vinay.badhwar@wvumedicine.org).

JTCVS Techniques 2021;8:95-9

2666-2507

Copyright (c) 2021 The Author(s). Published by Elsevier Inc. on behalf of The American Association for Thoracic Surgery. This is an open access article under the CC BY-NC-ND license (http://creativecommons.org/licenses/by-nc-nd/4.0/).

https://doi.org/10.1016/j.xjtc.2021.03.038
}

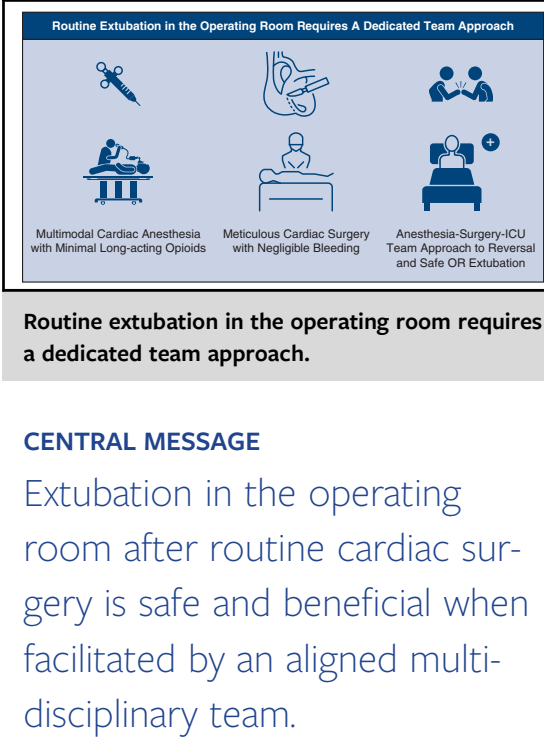

programs that perform OR extubations realize that it is applicable to far more than $5 \%$ of cardiac patients.

I am struck by 3 themes within this opinion piece that I find of particular importance. First, OR extubation in the appropriate patient is safe, and possibly beneficial. Second, the authors' emphasis on the multidisciplinary effort to accomplish the task should be lost on no one. The necessity to work together as a team of surgeons, anesthesiologists, perfusionists, and other, is appropriately highlighted. Finally, as the evidence continues to build that OR extubation in the right patient is an improvement in care, the authors challenge all of us to do more of it and lay out the tenets required to do so, safely. They should be commended. As we have done in the past (eg, with mitral valve repair), multiarterial grafting, transcatheter aortic valve replacements, and now, as trumpeted in this article, OR extubations, cardiac surgery should embrace every effort to improve what we do, regardless of its "inconvenience."

\section{Glenn J. R. Whitman, MD}

Prolonged intubation for $>24$ hours after cardiac surgery is associated with poor outcome and increased mortality. This may be largely a function of prolonged exposure to a combination of intravenous paralytics, sedation, and ventilatory support. The utility of wakefulness, active diaphragmatic contraction, and early ambulation have become 
predominant themes in intensive care applicable to management of the cardiac surgical patient in the immediate postoperative period. . $^{1-5-7}$ "Fast-track" or early extubation has been linked to improved quality and resource utilization. Admittedly, the definition of fast-track may be in the eye of the beholder, and it has been variably defined as endotracheal intubation for $<6$ to 12 hours postoperatively. ${ }^{2,3,6-8}$ The concept of early extubation originally faced considerable resistance when introduced $>2$ decades ago. ${ }^{7}$ Early opponents argued that the resultant effects of anesthesia produced too great a burden of hypercarbia and electrolyte imbalance to risk adding the loss of control of the airway to the already precarious equation. Nevertheless, the practice of rapid extubation has gained wide acceptance over the last decade, and it has become a significant enhancement to quality outcomes following cardiac surgery. ${ }^{1-4,6-8}$ Simultaneously, sporadic experience with safe extubation in the operating room (OR) following cardiac surgery was applied in a limited manner to off-pump coronary artery bypass grafting and selected operations performed on cardiopulmonary bypass, such as minimally invasive procedures. ${ }^{3,9,10}$ This has now been extended to patients following routine cardiac surgery, particularly those without significant coagulopathy and without intraoperative ventilatory or oxygenation aberrations with no anticipated difficulty in the use of mask ventilation or need for urgent reintubation. ${ }^{3,9-11}$ Today, there is growing support for safe routine extubation performed in the OR based on an increasing number of patient outcome benefits.

\section{CURRENT EVIDENCE}

Evidence to support OR extubation following routine cardiac surgery has grown through an extension of the experience with continuous quality improvement initiatives, early extubation protocols, and a cardiac anesthesia evolution away from historically opioid-dominant practices.

The Society of Thoracic Surgeons adopted extubation within 6 hours after surgery as a quality of care benchmark based on clear national registry information on beneficial impact. ${ }^{4}$ The goals for all early extubation protocols have been predicated on cost containment, efficient resource utilization, and value-based healthcare with a view to lowering opioid dosage. ${ }^{2,3,6-8}$ The use of and adherence to fast-track extubation protocols has most clearly attained these goals. ${ }^{1,3,6,8,11}$ Nevertheless, some have criticized that the 6-hour benchmark might not be based purely on physiologic evidence, as negative outcomes of operative mortality and morbidity may not significantly increase until patients have been intubated for $>12$ hours. $^{1}$ In a propensitymatched study of extubation after cardiac surgery using protocolized anesthetic management algorithms, OR extubation was compared to extubation in the intensive care unit (ICU) within 6 hours or 12 hours, and no significant differences in reintubation rates, morbidity, or mortality were found ${ }^{3}$; however, the frequency of direct-to-home discharge, overall length of stay, and cost containment were significantly enhanced in those extubated in the OR versus those extubated within 6 or 12 hours after ICU arrival. ${ }^{3}$ Furthermore, intubation beyond 12 hours is associated with demonstrable increases in morbidity, mortality, and length of stay. ${ }^{1,6,7}$ Although all postoperative complications cannot necessarily be attributed to prolonged ventilation, the potential increased risk of delirium and ventilator-associated pneumonia will always tilt the argument in favor of early extubation.

A growing appreciation of the potential injurious effect of barotrauma derived directly from high-tidal volume ventilation has resulted in the adoption of low-tidal volume algorithms predicated on protective volumes of 4 to $6 \mathrm{~mL} / \mathrm{kg} .{ }^{12}$ This is largely in keeping with the maintenance of diaphragmatic contraction and minimization of the reliance on and duration of neuromuscular blockade and related high-dose opioid-based anesthetics. Thus, the choice of sedatives has become an important consideration based on pharmacologic profiles, duration of action, and effect on hemodynamics both intraoperatively and during the recovery phase. As such, such medications as dexmedetomidine and ketamine have risen in prominence in view of their association with shorter time to extubation and shorter ICU length of stay compared with traditional agents such as propofol. ${ }^{13-15}$

The development and endorsement of clinical algorithms that support OR extubation require multidisciplinary engagement. ${ }^{3,9-11}$ For some patients, the benefit may be clearly superior to interval extubation, but for some more complex cases, the benefit may be noninferior. Choice of anesthetic and sedation, ventilatory management, and nursing staff and respiratory therapist availability all play important roles in maintaining commitment to the goal. ${ }^{11}$ Flynn and colleagues ${ }^{16}$ reported the utility of bedside huddles at the 4-hour mark postoperatively and its educational value provided to the intensivist, respiratory therapist, and nursing staff team. Goal-directed care may include the use of perioperative arterial blood gases and encouragement of the use of dexmedetomidine coupled with explicit reversal of the paralytic agents, each of which may serve as a useful adjunct to aid adherence to these policies. ${ }^{13-16}$ The same concepts hold for OR extubation. Accurate identification of appropriate patients is of great utility in clinical decision making at the bedside. Subramaniam and colleagues ${ }^{11}$ developed a prediction score based on age, body mass index, procedural factors (including emergent vs elective, full sternotomy vs nonfull, and type of cardiac surgery), as well as comorbidities (eg, chronic lung disease, diabetes, previous mediastinal radiation, peripheral vascular disease, hypoalbuminemia). An OR extubation score $>6$ was identified as a safe threshold for extubation in the OR following any form of adult cardiac surgery. ${ }^{11}$ 
It is this type of evidence that may aid the application of OR protocols to enhance the adoption of intraoperative extubation. To further promote adoption of alternative anesthetic techniques away from a high-dose opioid and muscle relaxant strategy, there has been a recent trend toward balanced anesthetic techniques including both multimodal therapy and regional anesthetic procedures for perioperative pain management. ${ }^{17,18}$ Although not mandatory, the potential inclusion of regional anesthesia may further facilitate OR extubation following cardiac surgery.

\section{MULTIDISCIPLINARY APPROACH}

The recent promotion and adoption of enhanced recovery after cardiac surgery pathways have fueled a growing interest in early extubation and OR extubation protocols. ${ }^{3,8}$ In tandem, there has been a rising popularity in the use of minimally invasive and robotic cardiac surgical techniques. These efforts are mirrored in the preferential choice of anesthetic techniques geared explicitly to the mitigation of prolonged intubation and length of stay while optimizing pain management using a multimodal approach. Indeed, there is a growing focus on responsible stewardship of constrained resources. In this vein, such practices as prehabilitation, minimally invasive surgical techniques for patients of higher comorbidity, modified cardiopulmonary bypass regimens, individualized anesthetic approaches, OR extubation, and dedicated physiotherapy regimens are fast becoming standard of care. ${ }^{3,8,10,11}$

The application of OR extubation extends beyond the mere use of minimally invasive operations. Similarly, the multidisciplinary approach extends beyond the mere selection of anesthetic agent. Through a team approach, extension of OR extubation may be applied to any operation that concludes in a routine manner without coagulopathy or risk of urgent reoperation. Intraoperative anesthetic algorithms may involve the use of a steroid as an antiemetic or an $\mathrm{H} 2$ blocker for ulceration prophylaxis, continuous monitoring of depth of anesthesia using bispectral index monitoring (with a target value of 40-50), and careful titration of neuromuscular blockade with complete reversal using such agents as sugammadex. Indeed, within our highvolume quaternary academic institution, this surgeryanesthesia collaborative team approach has enabled the extension of routine OR extubation to the majority of all adult cardiac operations performed. This includes redo operations, multivalve operations, operations in patients with abnormal ejection fraction, and even the occasional left ventricular assist device implantation or heart transplantation when appropriate criteria are met. Minimal opioids and benzodiazepines are used in induction, and neuromuscular blockade is typically avoided in the post-bypass period with any residual being reversed. During cardiopulmonary bypass, strict attention is paid to fluid balance, with careful selection of intravenous fluids and the judicious titration of vasopressors to maintain an appropriate mean arterial pressure. Retrograde autologous priming and routine hemofiltration are used to decrease the priming volume to maintain a zero-to-negative fluid balance. The patient is rewarmed to $36^{\circ} \mathrm{C}$ using the circuit, along with titrating the operating room temperature and forced air warmer, if necessary. From a surgical standpoint, meticulous technique and near-zero blood product use is practiced for all-comers regardless of complexity, and minimally invasive access incisions are preferred when feasible. Silicone-based chest tubes with optimal placement are favored to facilitate early postoperative mobility with the least amount of pain.

\section{POTENTIAL PITFALLS}

The benefits of early extubation are now well known. Nevertheless, careful mitigation strategies are necessary to prevent the deleterious effects of hypoxia and hypercarbia, which may compound the dysfunction of a patient with tenuous hemodynamics or right ventricular compromise. The compensatory catecholamine response may induce hypertension and pose a threat to fresh surgical anastomoses, as well as promote unwanted tachycardia. A mainstay of OR extubation is maintaining vigilance following extubation and resisting the temptation to focus only on the kinetics of transfer of care, such as moving the patient to the ICU bed, transferring monitors, and providing a sign-out regarding intraoperative management. After the patient is handed off in the ICU, it remains the shared responsibility of the anesthesia and critical care teams to closely monitor and intervene as the patient continues to emerge from anesthesia. Although very rare when OR extubation protocols and selection criteria are followed, ${ }^{11}$ there remains the potential risk of hypercarbia that may impair right ventricular function and may dictate the need for noninvasive positive pressure ventilation or, in exceedingly rare circumstances, reintubation. Thus, the constant emphasis on a team-based multistakeholder effort is essential. Programs without experienced postoperative critical care nurses, respirator therapies, or physicians available postoperatively may face challenges in implementing a team approach to OR extubation. Indeed, it is worth noting that a perception without evidence by any of the stakeholders (surgeons, anesthesiologists, intensivists, advanced practice professionals, nurses, and respiratory therapists) of potential patient harm may cultivate a reluctance to abide by evidence-based algorithms, and as such should be countered by open, inclusive dialogue that seeks to engender trust and collaboration on goal-driven care. ${ }^{3,6,8}$ This approach serves to shift the collective mindset toward a unified aim. Routine OR extubation is as much of a team mindset as a mechanism for quality improvement in cardiac surgery. 
Routine Extubation in the Operating Room Requires A Dedicated Team Approach

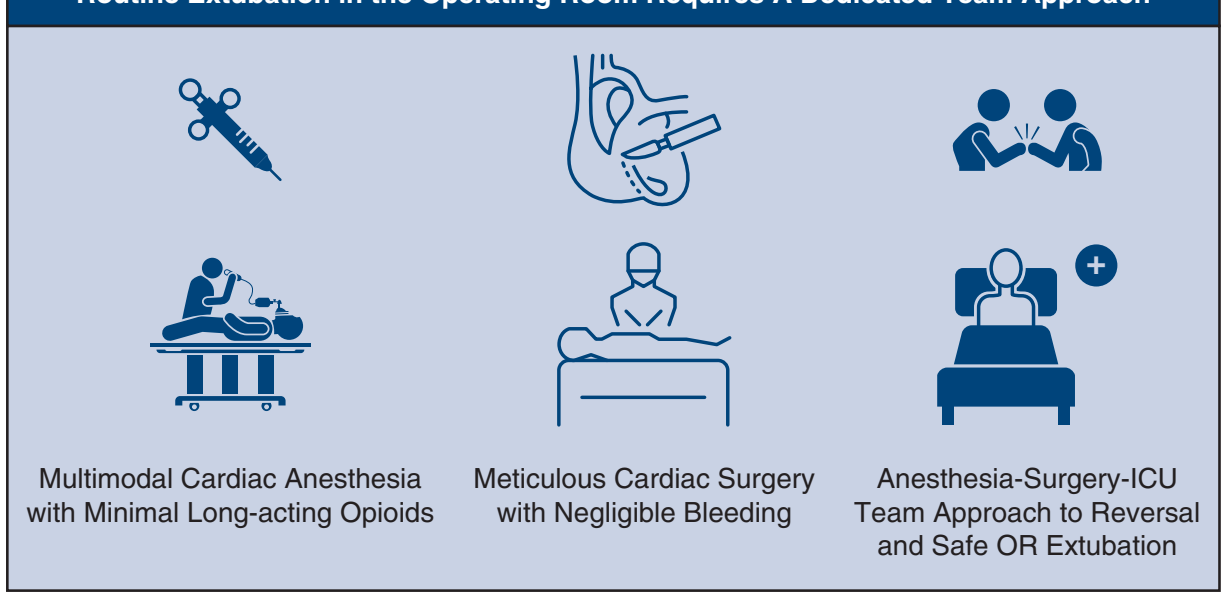

FIGURE 1. Routine extubation in the operating room requires a dedicated team approach. $I C U$, Intensive care unit; $O R$, operating room.

\section{PROGRAMMATIC CULTURE SHIFT}

The evolution of anesthetic technique to a low-opioid platform with multimodal analgesia is imperative to successful OR extubation. Decreasing opioid usage alone is inadequate, however; the anesthesia care team must be remain attentive to the use of muscle relaxants and anticipate timely cessation of volatile anesthetics and removal of the transesophageal echocardiography probe to permit early resumption of spontaneous ventilation, reversal of paralysis, and facilitate emergence soon after chest skin closure. Cooperatively, the technical precision of surgery must be maintained, and the need for surgical reexploration ideally should be kept to a minimum. The anesthesiologist may be less inclined to extubate in the face of concerns regarding the potential need for reexploration and the consequent risk of having to hastily reintubate a hemodynamically unstable cardiac patient. Similarly, the ICU team will need to grow comfortable managing an extubated cardiac surgical patient arriving to the ICU rather than one who is unconscious, sedated, and intubated. Clear and welldefined postoperative protocols are essential to continued success.

Once OR extubation has been established as a routine, the intubated patient arriving to the ICU will become a rarity. Nursing, allied health, respiratory therapy, and intensivists will all become aligned with OR extubation expectations and management protocols (Figure 1). Finally, and to the program's benefit, patients, their families, and their referring providers will come to expect this growing standard of quality care in cardiac surgery.

\section{CONCLUSIONS}

Extubation in the OR following routine cardiac surgery can be done safely with improved outcomes and greatly enhanced patient satisfaction. This true multidisciplinary effort challenges many of the institutional and traditional norms of the past. Perhaps initially inconvenient, the manifestation of the truth about OR extubation rests within its impact on programmatic quality and culture.

\section{Conflict of Interest Statement}

The authors reported no conflicts of interest.

The Journal policy requires editors and reviewers to disclose conflicts of interest and to decline handling or reviewing manuscripts for which they may have a conflict of interest. The editors and reviewers of this article have no conflicts of interest.

\section{References}

1. Crawford TC, Magruder JT, Grimm JC, Sciortino C, Conte JV, Kim BS, et al. Early extubation: a proposed new metric. Semin Thorac Cardiovasc Surg. 2016;28:290-9.

2. Cheng DC, Karski J, Peniston C, Raveendran G, Asokumar B, Carroll J, et al. Early tracheal extubation after coronary artery bypass graft surgery reduces costs and improves resource use. A prospective, randomized, controlled trial. Anesthesiology. 1996;85:1300-10.

3. Badhwar V, Esper S, Brooks M, Mulukutla S, Hardison R, Mallios D, et al. Extubating in the operating room after adult cardiac surgery safely improves outcomes and lowers costs. J Thorac Cardiovasc Surg. 2014;148:3101-9.e1.

4. Shahian DM, Jacobs JP, Badhwar V, Kurlansky PA, Furnary AP, Cleveland JC Jr, et al. The Society of Thoracic Surgeons 2018 adult cardiac surgery risk models: part 1-background, design considerations, and model development. Ann Thorac Surg. 2018:105:1411-8.

5. Gruther W, Benesch T, Zorn C, Paternostro-Sluga T, Quittan M, Fialka-Moser V, et al. Muscle wasting in intensive care patients: ultrasound observation of the M. quadriceps femoris muscle layer. J Rehabil Med. 2008;40:185-9.

6. Fitch ZW, Debesa O, Ohkuma R, Duquaine D, Steppan J, Schneider EB, et al. A protocol-driven approach to early extubation after heart surgery. J Thorac Cardiovasc Surg. 2014; 147:1344-50.

7. Trouillet JL, Combes A, Vaissier E, Luyt CE, Ouattara A, Pavie A, et al. Prolonged mechanical ventilation after cardiac surgery: outcome and predictors. $J$ Thorac Cardiovasc Surg. 2009;138:948-53.

8. Grant MC, Isada T, Ruzankin P, Whitman G, Lawton JS, Dodd -OJ, et al. Results from an enhanced recovery program for cardiac surgery. J Thorac Cardiovasc Surg. 2020;159:1393-402.e7.

9. Totonchi Z, Azarfarin R, Jafari L, Alizadeh Ghavidel A, Baharestani B, Alizadehasl A, et al. Feasibility of on-table extubation after cardiac surgery with cardiopulmonary bypass: a randomized clinical trial. Anesth Pain Med. 2018;8:e80158. 
10. Borracci RA, Ochoa G, Ingino CA, Lebus JM, Grimaldi SV, Gambetta MX. Routine operation theatre extubation after cardiac surgery in the elderly. Interact Cardiovasc Thorac Surg. 2016;22:627-32.

11. Subramaniam K, DeAndrade DS, Mandell DR, Althouse AD, Manmohan R, Esper SA, et al. Predictors of operating room extubation in adult cardiac surgery. J Thorac Cardiovasc Surg. 2017;154:1656-65.e2.

12. Güldner A, Kiss T, Serpa Neto A, Hemmes SN, Canet J, Spieth PM, et al. Intraoperative protective mechanical ventilation for prevention of postoperative pulmonary complications: a comprehensive review of the role of tidal volume, positive end-expiratory pressure, and lung recruitment maneuvers. Anesthesiology. 2015;123:692-713

13. Tsai CJ, Chu KS, Chen TI, Lu DV, Wang HM, Lu IC. A comparison of the effectiveness of dexmedetomidine versus propofol target-controlled infusion for sedation during fibreoptic nasotracheal intubation. Anaesthesia. 2010;65: 254-9.

14. Djaiani G, Silverton N, Fedorko L, Carroll J, Styra R, Rao V, et al. Dexmedetomidine versus propofol sedation reduces delirium after cardiac surgery: a randomized controlled trial. Anesthesiology. 2016;124:362-8.
15. Mazzeffi M, Johnson K, Paciullo C. Ketamine in adult cardiac surgery and the cardiac surgery intensive care unit: an evidence-based clinical review. Ann Card Anaesth. 2015;18:202-9.

16. Flynn BC, He J, Richey M, Wirtz K, Daon E. Early extubation without increased adverse events in high-risk cardiac surgical patients. Ann Thorac Surg. 2019;107: 453-9.

17. Macaire P, Ho N, Nguyen T, Nguyen B, Vu V, Quach C, et al. Ultrasound-guided continuous thoracic erector spinae plane block within an enhanced recovery program is associated with decreased opioid consumption and improved patien postoperative rehabilitation after open cardiac surgery-a patient-matched, controlled before-and-after study. J Cardiothorac Vasc Anesth. 2019;33:1659-67.

18. Krishna SN, Chauhan S, Bhoi D, Kaushal B, Hasija S, Sangdup T, et al. Bilateral erector spinae plane block for acute post-surgical pain in adult cardiac surgical patients: a randomized controlled trial. J Cardiothorac Vasc Anesth. 2019;33: 368-75.

Key Words: extubation, cardiac surgery, operating room 